\title{
A Fatal Presentation of Dermatomyositis
}

\author{
Khatiwoda $P_{,}^{1}$ Kayastha SR, ${ }^{2}$ Khatiwada $P,{ }^{1,2}$ Giri A, ${ }^{1,2}$ Baral $S,{ }^{2}$ Bista $D,{ }^{1,2}$ Pant $P^{1,2}$
}

${ }^{1}$ Richa Bajimaya Memorial Foundation

${ }^{2}$ Kathmandu University School of Medical Sciences (KUSMS)

\begin{abstract}
Dermatomyositis (DM) is an autoimmune disease that mainly affects the skin, muscle, and lung. The pathogenesis of skin inflammation in DM is not well understood. We present a 40-year-old male who presented with four months history of generalized skin lesion, pain and weakness. He had elevated transaminases with detectable muscle weakness. This case highlights the need to consider dermatomyositis with skin lesion and weakness; and the use of aggressive immunosuppressive therapies due to its associated vasculopathies.
\end{abstract}

\section{KEY WORDS}

Cutaneous lesions, dermatomyositis, idiopathic, myopathy, vasculitis

Citation

Khatiwoda P, Kayastha SR, Khatiwada P, Giri A, Baral S, Bista D et.al. A Fatal Presentation of Dermatomyositis. Kathmandu Univ Med $J$ 2012;39(3):78-82.

\section{INTRODUCTION}

Dermatomyositis (DM) is a chronic inflammatory disorder that can affect the skin, muscle, and other organs and is associated with significant morbidity and mortality. ${ }^{1-6}$ The prevalence of DM is not well-defined, as it is historically grouped together with polymyositis (PM) and inclusion body myositis (IBM) in most epidemiologic studies. ${ }^{7}$ Currently classified as an idiopathic inflammatory myopathy (IIM), much of the work in understanding DM has been focused on the muscle pathology that accompanies this disorder. ${ }^{8}$ The inflammatory mechanism is attributed to a primary T-helper-cell dependent, B-cell-mediated, local humoral immune mechanism, which causes vascular occlusion and capillary obliteration with ischemic change in the skin and muscle. ${ }^{4,9,10}$ Dermatomyositis may be associated with systemic manifestations including restrictive and interstitial lung disease and cardiomyopathy. ${ }^{4}$ We present a 40 -yearold male who presented with four months history of generalized skin lesion, pain and weakness.

\section{CASE REPORT}

A 40 years male from Devitar presented to Kathmandu University Hospital (Dhulikhel Hospital) in July 2011 for evaluation of generalized itching for six months; pain, weakness and skin lesions in different part of body for four months. The onset of the disease was acute, gradually progressive; he could eat with his hands but couldn't brush his teeth, could wear slippers but couldn't walk upstairs. This weakness amplified during the 15 days before his presentation. For the same duration gives history of scalp hair loss. He had some antibiotic medication for this, the nature of which could not be assessed. Although there was a history of subjective fever, there was no history of oral ulcers, shortness of breath, or recent immunizations. He was a farmer by occupation, led an active working lifestyle, non-smoker and occasional alcoholic. His medical and family history gave no abnormal findings.

At the time of admission, on examination, general condition 
Table 1. Laboratory values - at presentation, and discharge (after three weeks).

\begin{tabular}{|c|c|c|c|}
\hline & Normal Range & At presentation & At discharge \\
\hline Sodium & $134-143 \mathrm{mmol} / \mathrm{L}$ & 135 & 130 \\
\hline Potassium & $3.5-5.0 \mathrm{mmol} / \mathrm{L}$ & 4.2 & 4.1 \\
\hline Lactate dehydrogenase & 470-750 units/L & 6310 & - \\
\hline Aspartate aminotransferase (SGOT) & $15-40$ units/L & 137 & - \\
\hline Alanine aminotransferase (SGPT) & 10-55 units/L & 174 & - \\
\hline Alkaline phosphatase & 178-455 units/L & 127 & - \\
\hline Urea Nitrogen & $7-17 \mathrm{mg} / \mathrm{dL}$ & 36 & - \\
\hline Creatinine & $0.20-0.70 \mathrm{mg} / \mathrm{dL}$ & 0.6 & - \\
\hline Bilirubin total & $0.2-1.2 \mathrm{mg} / \mathrm{dL}$ & 1.2 & - \\
\hline Bilirubin direct & $0.1-0.2 \mathrm{mg} / \mathrm{dL}$ & 0.15 & - \\
\hline Serum glucose & $60-115 \mathrm{mg} / \mathrm{dL}$ & 63 & 74 \\
\hline Creatine phosphokinase (CPK) & $30-150$ units/L & $\begin{array}{l}1879 u / L \text { after } 4 \text { days of admission } \\
9980 u / L \text { after } 9 \text { days of admission } \\
17500 u / L \text { after } 16 \text { days of admission }\end{array}$ & \\
\hline Erythrocyte sedimentation rate & $0-13 \mathrm{~mm} / \mathrm{h}$ & 22 & - \\
\hline WBC counts (N-85\%,L-11\%,E-3\%) & $5.0-13.0 \times 10^{3} / \mathrm{mL}$ & 12.3 & 10.4 \\
\hline
\end{tabular}

was ill looking, height $-156.5 \mathrm{~cm}$, body weight $57.5 \mathrm{~kg}$, and body temperature $37.2^{\circ} \mathrm{C}$. blood pressure was $119 / 78$ $\mathrm{mmHg}$, pulse rate 73 per minute with a regular rhythm, and respiration 20 breaths per minute; liver palpable $5 \mathrm{~cm}$ below right sub-costal margin (RSCL). Cardio-pulmonary examinations were normal, neurological examination shows power on proximal muscle as $0 / 5$ whereas distal muscle showed $5 / 5$ both on upper and lower limbs. Sensation was intact, with normal bowel and bladder habit.

Skin changes include pigmentation over malar space, reticulate pigmentation over abdomen, poikiloderma over lower limbs, Guttron's sign, Shawl sign, Holster sign, vasculitis present over distal fingers, tenderness over digits. Typical erythematous lesion was not noticed in this patient, which could be because of the darker skin color of the patient.

Laboratory investigations are shown in table 1 . The abnormalities include increased CPK of $17500 \mathrm{u} / \mathrm{I}$ on 16th day of admission, raised Lactate dehydrogenase of 6310 units/L, elevated WBC count of $12.3 \times 10^{3} / \mathrm{mL}$ (N-85\%,L$11 \%, \mathrm{E}-3 \%)$, elevated Aspartate aminotransferase of 137 units/L and elevated Alanine aminotransferase (SGPT) of 174 Units/L. Ultrasonography presented fatty hepatomegaly. Muscle Biopsy indicated mild to moderate chronic inflammatory cells, presence of perivascular inflammatory cell, few atrophic and degenerative fibers suggesting active necrotizing myopathy, consistent with dermatomyositis.

However ANA and dsDNA were found to be negative. On third day of admission patient developed dysphagia but denied any nasogastric feed. He had no other neurological impairment. On the tenth day of admission he developed shortness of breath and fever with chest examination revealing crepitation over bilateral lung field.
He was treated with prednisolone $60 \mathrm{mg}$ initially then $80 \mathrm{mg}$. Antibiotics- Amoxiclav and metronidazole were used for respiratory tract infection. He also underwent physiotherapy and psychiatric consultation was done as the patient seemed as depressive.

By third week, patient was improving on his muscle weakness; with muscle power $3 / 5$ on proximal upper and lower limbs. The respiratory tract was also improving with decrease in crepitation. The patient was discharged on demand for Hindu festival of Dashain/Dashara. The patient was asked for follow but we were sad to hear that the patient expired after five days of being discharged from the hospital with the possible expected cause of death being aspiration pneumonia. Post mortem was not done, during this festival holiday.

\section{DISCUSSION}

Dermatomyositis (DM) is a systemic inflammatory disorder affecting the skeletal muscles, the skin, and other organs. ${ }^{11-13}$ The diagnosis of DM is still based on Bohan and Peter criteria: 1) symmetric proximal muscle weakness; 2) increased serum muscle enzymes; 3) myopathic changes upon electromyography; 4) typical histological findings on muscle biopsy; and 5) typical dermatologic manifestations, such as heliotrope rash or Gottron's papules. ${ }^{4,14}$ Diagnosed positive if any 4 out of 5 of the above criteria met, although latter criteria are most often used, they have several limitations (e.g., they do not include myositis-specific autoantibodies). We could meet four of the criteria for diagnosing presented case.

The estimated incidence of DM is approximately 1 per

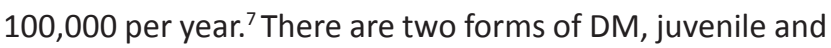
adult, those have overlapping but some distinct clinical features. ${ }^{7}$ There is a female to male predominance of about 


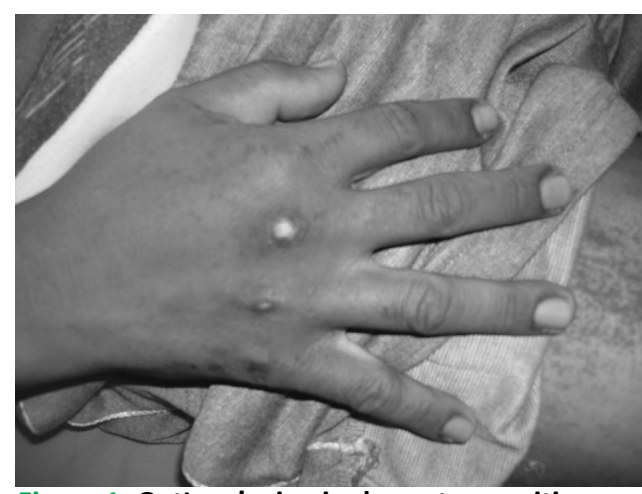

Figure 1. Gottron's sign in dermatomyositis.

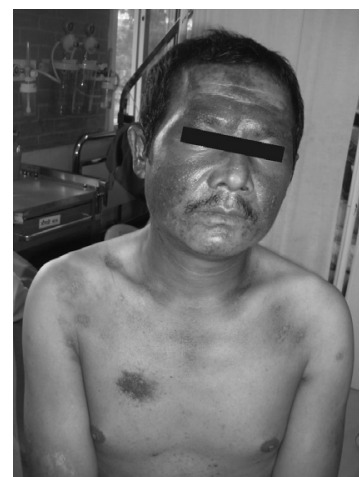

Figure 3. Facial erythema (erythroderma) in a patients with dermatomyositis.

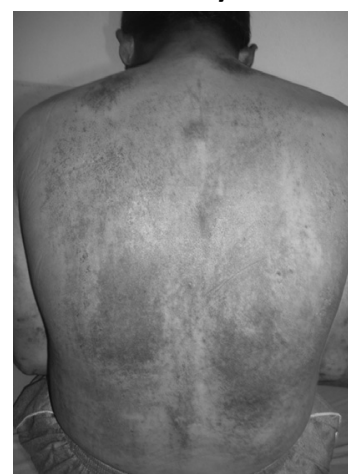

Figure 5. The shawl sign in dermatomyositis.

2:1. The peak incidence in adults occurs between the ages of 40 and 50 , but individuals of any age may be affected. ${ }^{15}$

Histologic features of DM include muscle fiber necrosis, degeneration and regeneration, and an inflammatory cell infiltrate. DM is considered to be a humorally mediated disorder in which the cellular infiltrate, located principally in perifascicular regions, is often focused around blood vessels. ${ }^{17-19}$ The terminal complement $\mathrm{C} 5 \mathrm{~b}-9$ membrane attack complex is detectable in vessel walls before the appearance of inflammatory cell infiltration in DM. This is absent in Polymyositis. The inflammatory infiltrate is composed of $B$ cells and plasmacytoid dendritic cells that are CD4+. Other typical features include perifascicular atrophy and fibrosis. Abnormal muscle fibers are usually grouped in one portion of the fascicle, suggestive of microinfarction mediated by blood vessel dysfunction. (refer cooment 12). The early activation of the complement cascade leads to the formation and deposition of the membranolytic attack complex on endomysial capillaries,

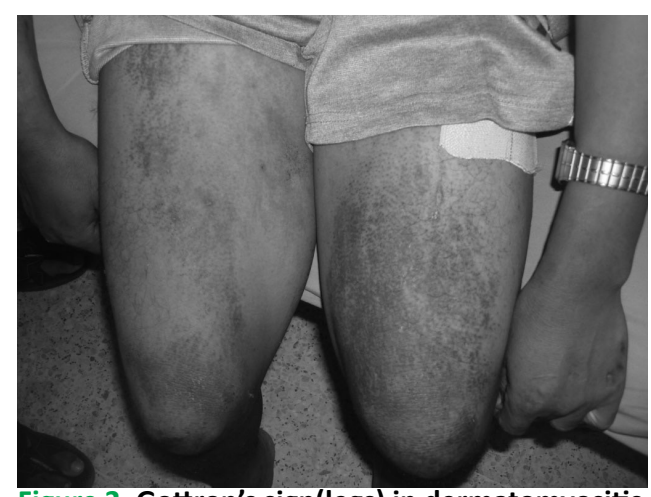

Figure 2. Gottron's sign(legs) in dermatomyositis.

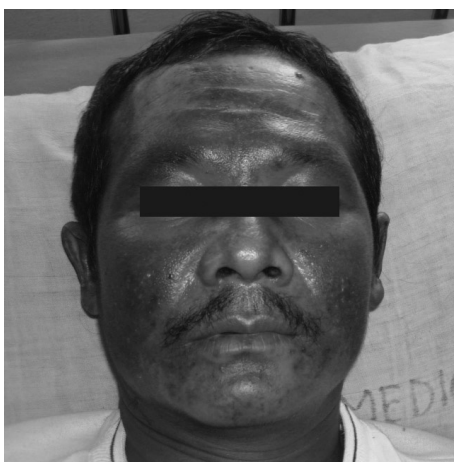

Figure 4. Heliotrope rash in dermatomyositis. resulting in their destruction and tissue ischemia. ${ }^{17,20}$

Although this key pathophysiologic event has strengthened the prospects for targeted immunotherapy in these conditions, the lack of identification of target auto antigens represents a major hurdle for the definition of specific immunotherapy in Dermatomyositis. Nevertheless, to date, nonspecific immunotherapeutic drugs have markedly improved the outcome of DM patients, and treatment does not selectively target pathogenic auto reactive T cells in PM or complement-mediated processes in DM.

Dermatomyositis is a multisystem disorder with a wide variety of potential clinical findings. Muscle weakness is the most common presenting feature of DM. The onset is usually insidious, with gradual worsening over a period of several months before medical attention is sought, very similar to this case presentation. The distribution of weakness is typically symmetric and proximal. An inability to swallow and symptoms of aspiration can reflect involvement of striated muscle in the pharynx or upper 
esophagus; dysphagia or dysphonia is generally associated with a rapidly progressive course. ${ }^{20,21}$ Several distinct rashes, generally present at the time of clinical presentation, occur in Dermatomyositis- Gottron's sign, Heliotrope rash, Shawl sign and $V$ sign, Erythroderma, Periungual abnormalities, Psoriasiform changes in scalp. DM may affect the lungs primarily, resulting in interstitial lung disease (ILD), which may lead to life threatening complications (i.e., ventilatory failure, secondary pulmonary arterial hypertension, or cor pulmonale)..$^{22}$

Esophageal motor activity disorders related to PM/DM may be responsible for swallowing dis- orders, including difficulty with solids and liquids, pain and pre-prandial discomfort in the sternal area, gastro esophageal reflux into the pharynx and/or mouth, coughing while eating, as well as life-threatening complications (e.g., aphagia to solids/liquids requiring total enteral feeding and aspiration pneumonia). ${ }^{12,21-24}$ Cardiac impairment may occur in up to $50 \%$ of patients, although it is more often asymptomatic in DM. ${ }^{1,25}$ Various abnormalities have been described, including mainly conduction defects and primarily endrhythm disturbances; congestive heart failure, pericarditis, and valvular disease are severe, less frequent cardiac complications in PM/DM patients. ${ }^{1,25}$ Evidence for an association between PM/DM and solid tumors came first from case reports, with the reported rate of cancer varying from $6 \%$ to $60 \% .^{1,12,23,26-30}$

Lab investigation shows raised muscle enzyme creatine kinase, lactate dehydrogenase, aldolase, and aminotransferases; and serological test can be ANA positive, Anti Jo positive. Autoantibodies are found in a majority of patients. Antinuclear antibodies in high titer suggest the presence of another connective tissue disease. Skin and/or muscle biopsies may establish the diagnosis in DM. Biopsy of a variety of DM skin findings, including Gottron's sign, the shawl sign, and erythroderma can provide confirmation of the diagnosis in the proper clinical setting. Histology of muscle shows perifascicular, perimysial, or perivascular infiltrates, perifascicular atrophy. MRI, chest X-ray, Barium

\section{REFERENCES}

1. Callen JP. Dermatomyositis. Lancet 2000;355:53-7.

2. Dalakas MC, Hohlfeld R. Polymyositis and dermatomyositis. Lancet 2003; 362:971.

3. Plotz PH, Dalakas M, Leff RL. Current concepts in the idiopathic inflammatory myopathies: polymyositis, dermatomyositis, and related disorders. Ann Intern Med 1989; 111:143.

4. Bohan A, Peter JB. Polymyositis and dermatomyositis (second of two parts). N Engl J Med 1975; 292:404-7.

5. Marie I. Morbidity and Mortality in Adult Polymyositis and Dermatomyositis. Curr Rheumatol Rep 2012. doi:10.1007/s11926012-0249-3.

6. N. Dwivedi. A fatal presentation of dermatomyositis with facial swelling. Int J Pediatr Otorhinolaryngol 2012. doi:10.1016/j. ijporl.2012.01.037. swallow can also be done, to see involvement of other organ system and MRI shows extent of damage in muscle.

Treatment for myopathy is done with high dose of corticosteroid. In our case, we started with $60 \mathrm{mg}$ of corticosteroid and later dose was increased to $80 \mathrm{mg}$. Alternative drugs that can be used are Azathioprine, Methotrexate, Mycophenolate mofetil, Monoclonal antiCD20 (Rituximab), Cyclophosphamide, Immunomodulation with Ivlg. Our strategy was slow change of treatment to Azathiorine, on follow up schedules. Treatment for skin lesion requires avoidance of sun exposure with immunosuppressant like Methotrexate, Mycophenolate mofetil, Hydroxychloroquine, and Chloroquine. Extra protein diet and observing the patient for development of complication is a factor to be considered in treatment.

\section{CONCLUSION}

Dermatomyositis is a rare disease entity. Prognosis depends on severity of myositis, presence of malignancy, cardiopulmonary system involvement. Patients severely affected at presentation or treated after long delays, those with severe dysphagia or respiratory difficulties, older patients, and those with associated cancer have a worse prognosis. The treatment of Dermatomyositis includes high-dose glucocorticoids in addition to disease-modifying agents or cytotoxic agents such as methotrexate. Most patients improve with therapy, and many make a full functional recovery, which is often sustained with maintenance therapy. Up to $30 \%$ may be left with some residual muscle weakness. Relapses may occur at any time. Early management of disease, diagnosis of any internal malignancy, any toxicity of treatment is important to look on follow up.

\section{ACKNOWLEDGEMENT}

Prof Dr TRS Bedi, Dr. Baburam Pokharel, Dr. Pankaj Pant, Dr Dharmendra Karn from Kathmandu University Teaching Hospital (Dhulikhel Hospital).

7. Mammen AL. Dermatomyositis and polymyositis: Clinical presentation, autoantibodies, and pathogenesis. Ann N Y Acad Sci 2010;1184: 134-53.

8. Greenberg SA, Amato AA. Uncertainties in the pathogenesis of adult dermatomyositis. Curr Opin Neurol 2004;17: 359-64.

9. Emslie-Smith AM, Engel AG. Microvascular changes in ear- ly and advanced dermatomyositis: a quantitative study. Ann Neurol 1990;27:343-56. doi:10.1002/ana.410270402

10. O'Hanlon TP, Miller FW. Genetic risk and protective factors for the idiopathic inflammatory myopathies. Curr Rheumatol Rep 2009;11:287-94.

11. Marie I, Hatron PY, Dominique S. Short term and long term outcomes of interstitial lung disease in polymyositis and dermatomyositis: a series of 107 patients. Arthritis Rheum 2011;63:3439-47. 
12. Marie I, Ménard JF, Hachulla E. Infectious complications in polymyositis and dermatomyositis: a series of 279 patients. Semin Arthritis Rheum 2011;41:48-60.

13. Marie I, Lahaxe L, Benveniste O. Long-term outcome of patients with polymyositis/dermatomyositis and anti-PM-Scl anti- body. $\mathrm{Br} J$ Dermatol 2010;162:337-44.

14. Bohan A, Peter JB. Polymyositis and dermatomyositis (first of two parts). N Engl J Med 1975; 292:403.

15. Bohan A, Peter JB, Bowman RL, Pearson CM. Computer-assisted analysis of 153 patients with polymyositis and dermatomyositis. Medicine (Baltimore) 1977; 56:255.

16. Tymms KE, Webb J. Dermatopolymyositis and other connective tissue diseases: a review of 105 cases. J Rheumatol 1985; 12:1140.

17. Kissel JT, Mendell JR, Rammohan KW. Microvascular deposition of complement membrane attack complex in dermatomyositis. $N$ Engl J Med 1986; 314:329.

18. Goebels N, Michaelis D, Engelhardt M. Differential expression of perforin in muscle-infiltrating $T$ cells in polymyositis and dermatomyositis. J Clin Invest 1996; 97:2905.

19. Hohlfeld R, Engel AG. The immunobiology of muscle. Immunol Today 1994; 15:269.

20. Dalakas MC. Immunotherapy of myositis: issues, concerns and future prospects. Nat Rev Rheumatol 2010;6:129-37.

21. Marie I, Hachulla E, Hatron PY, et al. Polymyositis and dermatomyositis: short term and longterm outcome, and predictive factors. J Rheumatol 2001;28:2230-7.
22. Marie I, Hatron PY, Hachulla E. Pulmonary involvement in polymyositis and in dermatomyositis. J Rheumatol 1998;25:1336-43.

23. Benbassat J, Geffel D, Larholt K. Prognostic factors in polymyositis/ dermatomyositis. A computer-assisted analysis of ninety-two cases. Arthritis Rheum. 1985;28:249-55.

24. Oh TH, Brumfield KA, Hoskin TL. Dysphagia in inflamma- tory myopathy: clinical characteristics, treatment strategies, and outcome in 62 patients. Mayo Clin Proc 2007;82:441-7.

25. Lie JT. Cardiac manifestations in polymyositis/dermatomyositis: how to get to heart of the matter. J Rheumatol 1995;22:809-11.

26. Schiopu E, Phillips K, Macdonald PM. Predictors of survival in a cohort of patients with polymyositis and dermatomyositis: effect of corticosteroids, methotrexate, and azathioprine. Arthritis Res Ther 2012;14:22.

27. Sultan SM, loannou Y, Moss K. Outcome in patients with idiopathic inflammatory myositis: morbidity and mortality. Rheu- matology (Oxford) 2002;41:22-6.

28. Torres C, Belmonte R, Carmona L. Survival, mortality and causes of death in inflammatory myopathies. Autoimmunity 2006;39:205-15.

29. Yamasaki Y, Yamada $H$, Ohkubo M. Longterm survival and associated risk factors in patients with adult-onset idio- pathic inflammatory myopathies and amyopathic dermatomyositis: experience in a single institute in Japan. J Rheumatol 2011;38:1636- 43.

30. Yu KH, Wu YJ, Kuo CF. Survival analysis of patients with dermatomyositis and polymyositis: analysis of 192 Chinese cases. Clin Rheumatol 2011;30:1595-601. 